RELATODE

EXPERIÊNCIA

\title{
Teste cardiopulmonar do exercício na prática clínica
}

\author{
Eva Cantalejo Munhoz Stadler de Souza, Adriana Cristina Bonat, Rosana Bento Radominski, \\ Ciro Romélio Rodriguez-Añez e Neiva Leite \\ Pontifícia Universidade Católica do Paraná (PUC) \\ Especialização em Ciência do Esporte e Medicina Desportiva
}

\section{RESUMO}

O teste cardiopulmonar do exercício (CPX) apresenta-se como uma metodologia de grande utilidade diagnóstica e prognóstica. O presente estudo teve por objetivo demonstrar que os dados obtidos em laboratório fora do ambiente hospitalar comportam-se como os dados descritos na literatura, com aplicabilidade na prática clínica em nosso meio. Metodologia: Trata-se de um relato de experiência, através da análise retrospectiva dos casos. O CPX foi realizado em condições de laboratório controladas, com bocal e clipe nasal, protocolo de rampa em esteira rolante e eletrocardiograma de 13 canais. Resultados: Entre os 261 testes, $53,3 \%$ eram em homens, idade média de 48,2 \pm 14,3 anos; ativos $(45,2 \%)$ ou sedentários $(34,5 \%)$. A capacidade aeróbia máxima foi superior e com declínio significativo para cada década de aumento na faixa etária entre os homens, enquanto nas mulheres o declínio significativo ocorreu entre os 30 e 60 anos. As mulheres apresentaram maior distribuição $(p=0,0006)$ nas classes funcionais "em programa de treinamento ou bem treinadas e motivadas". O consumo de oxigênio pico $\left(\dot{\mathrm{VO}}_{2}\right)$ foi significativamente superior nos testes máximos, mas o $\dot{\mathrm{VO}}_{2}$ do limiar anaeróbio ( $\dot{\mathrm{VO}}_{2} \mathrm{LA}$ ) não apresentou diferenças significativas, quando o teste obtido foi máximo ou submáximo. A capacidade funcional, avaliada pelo $\dot{\mathrm{V}} \mathrm{O}_{2} \mathrm{LA}$ como porcentagem do $\dot{\mathrm{VO}}_{2}$ máximo previsto, comparado à porcentagem do $\dot{\mathrm{VO}}_{2}$ máximo atingido, classificou mais indivíduos com compromisso circulatório $(p=0,002)$ ou com menor aptidão física em comparação com pacientes ativos ou em programa de treinamento $(\mathrm{p}<0,00001)$, exceto quando entre 50,0 e $59,0 \%$, em que o critério empregado não influenciou a classificação funcional $(\mathrm{p}=0,221)$. Não haver atingido $85,0 \%$

Recebido em: 7/7/2000

Aceito em: 31/10/2000

Endereço para correspondência:

Eva Cantalejo Munhoz Stadler de Souza

Rua Visconde do Rio Branco, 1.335, salas 93/94 e 101

80420-210 - Curitiba, Paraná - Brasil

E-mail: evacantalejo@uol.com.br

Rev Bras Med Esporte - Vol. 6, № 6 - Nov/ Dez, 2000 do $\dot{\mathrm{V}}_{2}$ máximo previsto foi a causa mais comum de anormalidade, mais freqüente e significativo entre as mulheres. Conclusão: Os dados obtidos são comparáveis aos descritos na literatura, sugerindo que o CPX é uma metodologia factível, que poderia ser empregada rotineiramente na prática clínica em nosso meio.

Palavras-chave: Ergoespirometria. Teste cardiopulmonar do exercício ou prova ergométrica multiparamétrica.

\section{ABSTRACT}

\section{Cardiopulmonary stress testing in clinical practice}

Cardiopulmonary stress testing (CPT) is a very useful tool to determine the diagnosis and prognosis in clinical practice. The objective of this study is to demonstrate that data obtained in a laboratory outside the hospital are similar to those described in the literature. Methodology: Patients were submitted to CPT, treadmill ramp protocol, and 13 lead electrocardiogram to evaluate CPT in the clinical practice. Results: Among 261 CPT, 53.3\% were male, mean age 48.2 \pm 14.3 years, with active $(45.2 \%)$ or sedentary (34.5\%) lifestyle. Male patients showed higher maximal aerobic capacity $\left(\dot{\mathrm{V}} \mathrm{O}_{2}\right.$ max) and a significant decrease of $\dot{\mathrm{V}} \mathrm{O}_{2}$ for each ten years of increment in age, but it decreased from 30 to 69 years in females. Females showed a significant higher $(p=0.0006)$ distribution in functional classes described as "in training programs or well trained and high motivation". A $\dot{\mathrm{V}} \mathrm{O}_{2}$ max was superior in maximal effort tests, but anaerobic threshold ( $\left.\mathrm{VO}_{2} A T\right)$ did not show differences between maximal or submaximal tests. The functional capacity evaluated by $\dot{V} \mathrm{O}_{2} A T$ as a fraction (\%) of the $\dot{V} \mathrm{O}_{2}$ max predicted in comparison to the fraction of the $\dot{V} \mathrm{O}_{2}$ max measured was more rigorous, which implies a higher number of patients with circulatory impairment ( $p$ $=0.002$ ) and also with lower physical capacity as opposed to active patients or patients under training programs ( $p<$ 0.00001); however, between 50.0\% and 59.0\% of the patients could be classified equally by one or the other criteria $(p=0.221)$. The more frequent abnormality in CPT was that it did not achieve $85.0 \%$ of the $\dot{\mathrm{V}} \mathrm{O}_{2}$ max predicted, 
more significant for females. Conclusion: Despite the limitations, this experience indicates that CPT data are reproducible in a laboratory outside the hospital, suggesting that CPT may be applied in clinical practice.

Key words: Spirometry. Cardiopulmonary exercise testing. Spiroergometry.

\section{INTRODUÇÃO}

O teste cardiopulmonar do exercício é um método que ocupa lugar definido na Medicina, particularmente na Cardiologia, na Medicina Interna, na Pneumologia e na Medicina Esportiva. $\mathrm{O}$ emprego da metodologia vem-se tornando cada vez mais fácil graças à simplificação dos equipamentos disponíveis, bem como à redução dos custos, melhor precisão e calibração confiável. Destaca-se ainda o acoplamento da informática aos mesmos, que permite a análise simultânea de múltiplos dados em tempo real e, com o advento da oximetria de pulso, a coleta de sangue arterial torna-se dispensável em muitos casos, o que facilita o seu uso clínico ${ }^{1-9}$.

Além da sua aplicação na rotina para o diagnostico diferencial das doenças pulmonares com as cardíacas ${ }^{10-15}$, a partir dos estudos de Mancini et al. ${ }^{16}$ tornou-se ferramenta útil para a seleção dos candidatos a transplante cardíaco ${ }^{1,5-}$ 7,11,13-15,17. Com o desenvolvimento dos esportes competitivos e a população de atletas de alto rendimento passou também a ser amplamente empregado na Medicina Esportiva, com todas as implicações do seu aspecto multidiscipli$\operatorname{nar}^{17,18}$.

O teste cardiopulmonar do exercício para o diagnóstico em populações específicas vem sendo utilizado desde a década de 70-80 em nosso meio ${ }^{19-21}$, mas apenas recentemente um único estudo randomizado, publicado por $\mathrm{Ne}$ der et al., avaliou os valores médios na população de São Paulo 22 .

\section{OBJETIVOS}

O presente estudo teve por objetivos: demonstrar que os dados obtidos em laboratório localizado fora de ambiente hospitalar e de pesquisa comportam-se como os descritos na literatura, com aplicabilidade na prática clínica em nosso meio, e avaliar a classificação funcional proposta por Zavala e Mazzei.

\section{CASUÍSTICA E MÉTODOS}

Trata-se de um relato de experiência, através da análise retrospectiva de casos. No momento da coleta dos dados não estava disponível na literatura nenhum dado populacional brasileiro, razão pela qual foram empregados os va- lores previstos, ajustados para sexo, idade e composição corporal, publicados por Wasserman et al., amplamente empregados em estudos prévios ${ }^{3}$.

Todos os pacientes executaram exercício com protocolo de rampa individualizado, em esteira (KT 10200 - Inbramed - Porto Alegre, Brasil) e os dados foram armazenados no software Elite (Micromed Biotecnologia, Brasília, Brasil). O analisador metabólico utilizado foi o TEEM 100, sistema de espirometria de circuito aberto (AeroSport; Ann Arbor, Michigan, USA - InbraSport - Inbramed - Porto Alegre, Brasil), com seus acessórios, previamente validado por Novitsky et al. ${ }^{23}$ e Wideman et al. ${ }^{24}$. O bocal e o clipe nasal foram empregados em todos os casos, com uma calibração obrigatória, realizada antes de cada exame.

Os dados ventilatórios foram obtidos em tempo real, mas o intervalo da amostra selecionado para todos os pacientes foi de 20 segundos e, a partir das medidas diretas, originaram cálculos derivados, gráficos, tabelas e curvas. O consumo máximo de oxigênio atingido $\left(\mathrm{V}_{2}\right.$ máximo $)$ foi considerado como o $\dot{\mathrm{VO}}_{2}$ pico. O limiar anaeróbico foi determinado pelos critérios de Wasserman et al. ${ }^{3}$ e Beaver et $a l .{ }^{25}$, por um único observador, no momento do laudo final do teste, sem revisão posterior. A oximetria de pulso foi obtida com o oxímetro de pulso Ohmeda Biox 3700, através de um dispositivo descartável conectado ao dedo.

Os pacientes foram classificados segundo a capacidade funcional, empregando o consumo de oxigênio do limiar anaeróbico como porcentagem do consumo máximo de oxigênio atingido para comparação com o sugerido por Zavala e Mazzei $^{7}$ (como porcentagem do consumo máximo de oxigênio previsto) em: menor que $40,0 \%$ - comprometimento circulatório; entre $40,0 \%$ e $49,0 \%$ - inatividade física; entre 50,0\% e 59,0\% - atividade física; entre 60,0\% e 79,0\% - indivíduo em programa de treinamento; maior ou igual a $80,0 \%$ - indivíduo bem treinado e motivado.

A pressão arterial foi obtida através de um esfigmomanômetro de mercúrio fixado na parede e com o manguito de tamanho adequado para a circunferência do braço do paciente.

Todos os pacientes foram informados e instruídos sobre o procedimento a ser executado, desconfortos e problemas durante a execução do teste, e encorajados a realizarem um esforço máximo, sintoma limitado, com demonstração do uso do bocal, possibilidades de comunicação durante o exercício (através de sinais manuais, quadros ilustrativos e a Escala de Percepção do Esforço de Borg ${ }^{26}$ ) e ainda a proibição total de inspirar ou expirar fora do sistema. Todos os pacientes assinaram um termo de consentimento para a realização do teste (anexo). Os sinais, sintomas e demais intercorrências foram amplamente detalhados e discutidos com o paciente após o término do teste. A preparação do 
paciente, os registros e a interpretação do eletrocardiograma de esforço (de 13 canais) seguiram orientações dos autores e, ainda, rigorosamente os Consensos Brasileiro de Ergometria e Americano de Cardiologia ${ }^{4,27,28,29}$.

$\mathrm{O}$ teste de esforço foi considerado máximo quando o paciente atingiu uma razão de resposta ventilatória igual ou superior a 1,09 .

\section{Termo de Consentimento para o \\ Teste Cardiopulmonar do Exercício}

1) Farei uma prova de esforço em esteira rolante ou bicicleta ergométrica para diag nosticar meu estado físico e a natureza e medida de qualquer problema circulató. rio, pulmonar ou cardíaco.

2) Os equipamentos a serem empregados serão mostrados a mim antes do início do teste.

3) A prova verificará 0 ar (oxigênio e dióxido de carbono) que sai dos meus pulmões enquanto pedalo ou caminho na esteira. Também um monitor eletrocardiográfico indicará ao médico examinador os batimentos do meu coração; e minha pressão arterial será medida freqüentemente; um aparelhinho será aderido à ponta de um dos meus dedos para registrar a saturação de oxigênio do meu sangue.

4) Respirarei por meio de uma máscara ou bocal, que será colocado entre os lábios e os dentes, enquanto realizar 0 exercício e também será colocado um clipe nasal, para impedir que 0 ar escape pelos orifícios do meu nariz.

5) 0 médico examinador poderá decidir extrair amostras de sangue para análise, por meio de um "escalpe" que seria inserido em minha artéria, mas também devo concordar com este item do exame separadamente.

6) Estou informado de que não existe nenhum risco na medição da respiração e muito pouco na prova de exercício. Se sofro de uma doença cardíaca, a prova poderia causar "dor no peito" ou batimentos irregulares do coração. A presença do médico ao meu lado, controlando minha pressão sanguínea, observando 0 monitor eletrocardiográfico e seguindo a saturação de oxigênio no sangue, fará com que estes riscos sejam mínimos. Se tiver dor no peito, batimentos irregula. res, tonturas, diminuição da pressão arterial ou do oxigênio no sangue, 0 médico procederá de acordo com as minhas necessidades clínicas.

7) Durante 0 exercício, a carga de trabalho será aumentada de forma progressiva, lentamente, até onde eu possa tolerar. Por outro lado, posso solicitar o término do exame em qualquer momento em que não me sinta bem e 0 médico também poderá interromper a prova, por critérios médicos.

8) Depois do exercício, sentirei cansaço físico, com os músculos muito cansados e até podem estar doloridos, porque a proposta inicial será realizar 0 meu máximo esforço, que naturalmente será desconfortável, quanto ao cansaço.

9) Entendo perfeitamente qual o objetivo deste teste e sei também como ele será realizado, porque será explicado em detalhes e tenho conhecimento dos seus riscos.

10) Estou de acordo em fazer a Prova de Esforço ou o Teste Cardiopulmonar do Exercício no Laboratório de Exercício sob a responsabilidade da Dra. Eva Cantalejo Munhoz - CRM/PR 9946, localizado na Rua Visconde do Rio Branco, 1.335 - salas 93 e 94 (nono andar) e, estou ciente de que poderei compor estudos clínicos, mantendo entretanto os princípios de ética que regem a relação médico-paciente, estabelecidos pelo Conselho Regional e Federal de Medicina.

Data:...............................

Nome do paciente:

Assinatura do paciente:

Nome do médico: Eva Cantalejo Munhoz

Assinatura do médico:
Uma vez obtidos e interpretados os dados, uma descrição detalhada no formato de laudo foi elaborada e os dados foram transferidos para uma planilha eletrônica do software Excel, especialmente elaborada para armazenar o banco de dados.

\section{Análise estatística dos dados}

Para a análise estatística dos dados foi empregada a análise descritiva dos dados através de tabelas e gráficos. Foram utilizados os testes comparação entre duas proporções, paramétrico $t$ de Student (ambos pelo software Primer of Biostatistics) e os não-paramétricos qui-quadrado, com correção de Yates e teste exato de Fisher (pelo software Epi-Info) para amostras independentes. O nível de significância (ou probabilidade de significância) mínimo adotado foi de $5,0 \%(\mathrm{p}<0,05)$.

\section{RESULTADOS}

\section{Características gerais}

A população em estudo compreende 261 pacientes consecutivos, sendo que pacientes do sexo masculino representaram 53,3\% da amostra. A idade variou entre 16 e 78 anos (48,2 $\pm 14,3$ anos), sendo no sexo masculino 48,9 \pm 15,0 e feminino $47,5 \pm 13,5$. Distribuídos em faixas etárias por décadas, a maioria dos pacientes encontravam-se na faixa etária entre 50 e 59 anos $(29,1 \%)$, sendo maior a distribuição dos pacientes do sexo masculino nesta faixa etária $(32,4 \%)$. Pacientes do sexo feminino apresentaram uma maior distribuição na faixa etária entre 40 e 49 anos $(27,0 \%)$.

Quanto ao nível de atividade física diária, a maioria dos pacientes eram apenas ativos $(45,2 \%)$ ou sedentários $(34,5 \%)$. Pacientes portadores de cardiopatias representaram $18,8 \%$ da amostra e apenas quatro pacientes $(1,5 \%)$ eram atletas.

\section{Indicações clínicas}

Entre os 261 pacientes, $66,3 \%$ apresentavam-se assintomáticos no momento do teste e $33,7 \%$ deles receberam indicação para o teste para definição diagnóstica, por sintomas. Entre as indicações, a avaliação cardiovascular foi responsável por $28,4 \%$, sendo mais freqüente no sexo masculino $(33,1 \%)$, mas sem significância estatística; seguida por dor precordial $(23,8 \%)$, maior para o sexo feminino $(30,3 \% \times 18,0 \%)(\mathrm{p}=0,029)$; insuficiência coronária $(18,8 \%)$ significativa para o sexo masculino $(26,6 \%$ x 9,8\%) ( $p<0,0001)$ e fadiga $(11,9 \%)$, com diferença significativa para o sexo feminino $(18,0 \%$ x $6,5 \%)(\mathrm{p}=0,008)$. Outras indicações clínicas foram: dispnéia $(8,4 \%)$; hipertensão arterial sistêmica $(8,0 \%)$; doença valvular $(7,7 \%)$; palpitações $(3,8 \%)$. 


\section{Dados obtidos das trocas ventilatórias}

Teste de esforço máximo ( $R R \geq 1,09$ ) foi obtido em $75,9 \%$ dos pacientes, sendo sintoma limitado (fadiga) em $98,0 \%$.

$\mathrm{O}$ consumo de oxigênio atingido $\left(\mathrm{V}_{2}\right.$ máximo atingido) médio foi $31,3 \pm 8,8 \mathrm{ml} /(\mathrm{kg} \cdot \mathrm{min})$ correspondendo à média do consumo de oxigênio máximo previsto de $88,4 \%$ $\pm 17,0 \%$, sendo maior para o grupo masculino em comparação com o grupo feminino: $34,4 \pm 9,6 \mathrm{ml} /(\mathrm{kg} \cdot \mathrm{min}) \times 27,9$ $\pm 7,2 \mathrm{ml} /(\mathrm{kg} \cdot \mathrm{min})$ ou $94,1 \% \pm 17,9 \% \times 82,1 \% \pm 13,4 \%(\mathrm{p}<$ $0,0001)$.

$\mathrm{O}$ consumo de oxigênio do limiar anaeróbio ( $\left.\mathrm{V}_{2} \mathrm{LA}\right)$ apresentou valores médios superiores no sexo masculino $(21,6 \pm 6,2 \times 19,7 \pm 4,7 \mathrm{ml} /(\mathrm{kg} \cdot \mathrm{min})(\mathrm{p}=0,004)$, mas o $\dot{\mathrm{V}}_{2} \mathrm{LA}$, expresso como porcentagem do $\dot{\mathrm{VO}}_{2}$ máximo atingido, apresentou média superior no sexo feminino $(71,4 \%$ $\pm 11,1 \% \times 64,2 \% \pm 12,0 \% ; \mathrm{p}<0,0001)$.

O pulso de oxigênio $\left(\dot{\mathrm{VO}}_{2} / \mathrm{FC}\right)$ foi de $12,8 \pm 4,0 \mathrm{ml} / \mathrm{bati}-$ mento, correspondendo a $90,2 \% \pm 17,0 \%$ do pulso de oxigênio previsto; menor para as mulheres $(9,9 \pm 1,9 \times 15,4 \pm$ $3,5 \mathrm{ml} /$ batimento $)$ ou $(83,6 \% \pm 13,6 \% \times 96,2 \% \pm 17,5 \% ; \mathrm{p}$ $<0,0001)$.

Apenas $9,1 \%$ reproduziram sintomas durante o teste. A dor precordial foi o sintoma mais frequiente $(5,7 \%)$. Embora mais freqüentemente entre as mulheres em comparação com os homens $(6,1 \%$ x 5,1\%), não houve significância estatística $(\mathrm{p}=0,4915)$.

\section{Capacidade aeróbica}

$\mathrm{O}$ consumo máximo de oxigênio $\left(\mathrm{V}_{2}\right.$ pico atingido) médio foi maior para o sexo masculino $(34,4 \pm 9,6 \times 27,9$ $\pm 6,4 \mathrm{ml} /(\mathrm{kg} \cdot \mathrm{min})(\mathrm{p}<0,0001)$. Apresentou redução para cada década de aumento na faixa etária, com significância estatística para o sexo feminino nas faixas etárias de 30-39 anos em comparação com a faixa etária 40-49 anos ( $\mathrm{p}=$ 0,008), de 40-49 anos em comparação com a de 50-59 anos $(\mathrm{p}=0,036)$ e na de 50-59 anos em comparação com a de 60-69 anos $(p=0,049)$. Pacientes do sexo masculino apresentaram decréscimo significativo em todas as faixas etárias, exceto quando comparados 50-59 anos com 60-69 anos $(\mathrm{p}=0,246)$ (gráfico 1).

\section{Teste máximo x teste submáximo}

Entre os 261 pacientes, 198 (75,9\%) executaram um teste de esforço considerado máximo ( $R R \geq 1,09$ ), sintoma limitado, sendo por fadiga em 194 pacientes e nos outros quatro, o esforço foi suspenso; taquicardia ventricular não sustentada repetitiva, taquicardia paroxística supraventricular, dispnéia e claudicação. Entre os 63 pacientes $(24,1 \%)$ com testes considerados submáximos (RR < 1,09), 62 apresentaram fadiga e um paciente solicitou término do exercí-

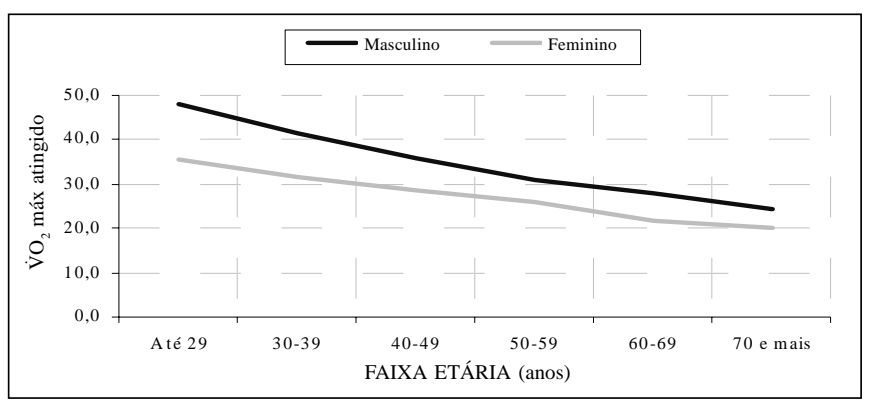

Gráfico 1 - Redução do consumo máximo de oxigênio por faixa etária e sexo

cio por dor lombar. A fadiga motivou o término do exercício, independente do teste obtido haver sido máximo ou submáximo $(\mathrm{p}=0,6511)$.

$\mathrm{O} \dot{\mathrm{VO}}_{2}$ máximo relativo atingido foi significativamente maior ( $\mathrm{p}<0,0001$ ), nos testes máximos em comparação com os testes submáximos $(32,0 \pm 8,6 \times 29,2 \pm 9,3 \mathrm{ml} /$ (kg.min). Esta diferença não foi observada quando analisado o $\dot{\mathrm{V}}_{2}$ máximo atingido como porcentagem do $\dot{\mathrm{VO}}_{2}$ máximo previsto $(88,4 \% \pm 16,9 \%$ x $88,5 \% \pm 17,4 \%$, para testes máximos e submáximos, respectivamente; $\mathrm{p}=0,747$ ).

O consumo de oxigênio do limiar anaeróbio foi determinado em todos os testes e não apresentou diferenças estatísticas quando o teste foi máximo ou submáximo (20,7 $\pm 5,8 \times 20,7 \pm 4,9 \mathrm{ml} /(\mathrm{kg} \cdot \mathrm{min}) ; \mathrm{p}=1,000)$. Expresso como porcentagem do $\mathrm{V}_{2}$ máximo atingido, foi superior nos testes submáximos $(72,9 \% \pm 13,1 \%$ x $65,9 \% \pm 11,3 \%$; $\mathrm{p}<$ $0,0001)$. Quando analisado o $\dot{\mathrm{VO}}_{2} \mathrm{LA}$ como porcentagem do $\dot{\mathrm{VO}}_{2}$ máximo previsto, também foi superior nos testes submáximos $(63,5 \% \pm 13,4 \%$ x 58,0\% $\pm 13,1 \%$; $(\mathrm{p}<$ 0,0001).

\section{Capacidade funcional}

Empregando o consumo de oxigênio do limiar anaeróbio como porcentagem do consumo máximo de oxigênio previsto, não houve diferenças significativas entre homens e mulheres. A maioria dos pacientes foram classificados como em atividade física ou em programa de treinamento $(67,4 \%)$; inatividade física $(20,2 \%)$, indivíduos bem treinados e motivados $(7,0 \%)$ e pacientes com comprometimento circulatório $(5,4 \%)$.

Empregando o consumo de oxigênio como porcentagem do $\dot{\mathrm{VO}}_{2}$ máximo atingido, a maioria dos pacientes $(78,3 \%)$ foram classificados como em atividade física ou em programa de treinamento, com significância estatística comparada à forma acima descrita $(\mathrm{p}<0,00001)$, e apenas $0,4 \%$ dos pacientes foram classificados como comprometimento circulatório, também com significância estatística ( $\mathrm{p}=$ 0,002 ). Quando comparadas as duas formas empregadas para obter a classificação funcional, houve diferença esta- 


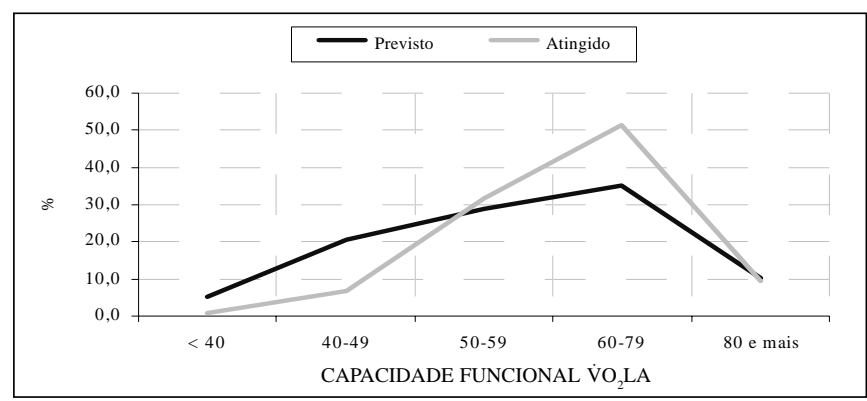

Gráfico 2 - Capacidade funcional $\dot{V} O_{2} L A$ (previsto x atingido) - sexo masculino

tisticamente significativa em todas as faixas etárias, exceto nos pacientes que seriam classificados como atividade física ( $\dot{\mathrm{V}} \mathrm{O}_{2} \mathrm{LA}$ entre $50,0 \%$ e $59,0 \%$ ), que poderiam haver sido classificados das duas formas e sem diferenças estatísticas ( $\mathrm{p}=0,221)$.

Pacientes do sexo feminino apresentaram maior distribuição na classificação funcional como em programa de treinamento ou bem treinadas e motivadas $(81,2 \%$ x $61,1 \%$; $\mathrm{p}=0,0006$ ) (gráficos 2 e 3 ).

\section{Resultado do teste}

Os testes foram classificados como anormais em 97 pacientes. A causa mais comum de anormalidade foi não haver atingido $85,0 \%$ do consumo máximo e oxigênio previsto, mais frequiente entre as mulheres $(91,5 \%$ x $73,7 \%$; $p$ $=0,038$ ), seguida da isquemia miocárdica, significativa para os pacientes do sexo masculino $(44,7 \%$ x $20,3 \%$; $\mathrm{p}=0,019)$.

\section{DISCUSSÃO}

Pacientes do sexo feminino tiveram dor precordial, como maior indicação clínica para o teste $(\mathrm{p}=0,029)$ e fadiga $(\mathrm{p}$ $=0,008$ ) em comparação com os pacientes masculinos. Os homens apresentaram indicação mais freqüente para avaliação cardiovascular, mas com o diagnóstico mais freqüente de insuficiência coronária $(\mathrm{p}<0,0001)$, possivelmente porque já apresentavam doenças e se encontravam em controle clínico e, também, porque sempre foram considerados de maior risco para as doenças cardiovasculares.

Apenas $9,1 \%$ dos pacientes reproduziram sintomas durante o teste e, coincidentemente, o sintoma mais freqüente foi dor precordial; apesar de mais freqüente nas mulheres, não apresentou diferenças estatísticas, corroborando os achados de que a reprodutibilidade dos sintomas durante um teste de esforço é pobre, mas quando presente constitui-se em um achado relevante ${ }^{4,27,30}$.

A capacidade aeróbica foi significativamente superior nos pacientes do sexo masculino em todas as idades, mas as mulheres apresentam classe funcional superior à dos homens com maior distribuição em programa de treina-

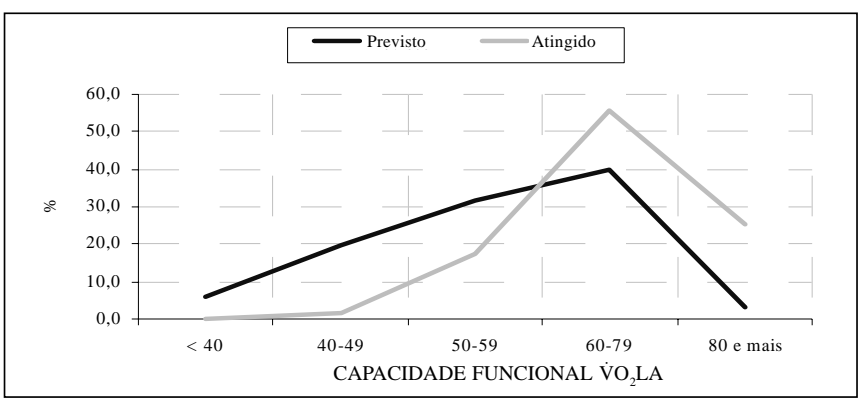

Gráfico 3 - Capacidade funcional $\dot{V} O_{2} L A$ (previsto x atingido) - sexo feminino

mento ou bem treinada e motivada $(\mathrm{p}=0,0006)$. Na redução do consumo máximo de oxigênio com o aumento de cada década de faixa etária examinada, tanto nos homens como nas mulheres, a significância para as mulheres não foi alcançada apenas nos extremos das faixas etárias (até 29 anos e $\geq 70$ anos). Entre os homens, a significância ocorreu em todas as faixas etárias, exceto entre 50-59 e 60-69 anos, sugerindo um comportamento do consumo máximo de oxigênio similar entre os 50 e 69 anos, provavelmente influenciado pela amostra. $\mathrm{O}$ achado de $\dot{\mathrm{VO}}_{2} \mathrm{LA}$ superior $\left(63,5 \pm 13,4 \%\right.$ x $58,0 \pm 13,1 \%$ do $\dot{\mathrm{VO}}_{2}$ máximo previsto; $\mathrm{p}$ $<0,0001)$ nos testes submáximos $(\mathrm{RR}<1,09)$ sugere que a habilidade em realizar esforço máximo pode estar mais relacionada ao hábito do exercício (treinamento), que à capacidade aeróbica máxima, uma vez que o $\dot{\mathrm{V}}_{2}$ máximo atingido foi superior nos testes considerados máximos (RR $\geq 1,09),(32,0 \pm 8,6 \times 29,2 \pm 9,3 \mathrm{ml} / \geq \mathrm{kg} \cdot \mathrm{min}) ; \mathrm{p}<0,0001$. Ainda, a atividade física poderia apresentar-se como fator importante na motivação dos pacientes para a tolerância aos desconfortos necessários para atingir o máximo esforço durante um teste de laboratório ${ }^{3,4,6-8,31-33}$.

A classificação funcional segundo os critérios de Zavala e Mazzei ${ }^{7}$ apresentou distribuição semelhante entre os pacientes de ambos os sexos.

Os autores compararam a classificação funcional empregando o consumo de oxigênio do limiar anaeróbio como porcentagem do consumo máximo de oxigênio atingido em comparação como porcentagem do consumo máximo de oxigênio previsto ${ }^{7}$ e observaram que seriam excluídos pacientes com comprometimento circulatório (de 5,4\% para $0,4 \% ; p=0,002)$. Parece óbvio porque no primeiro caso o indivíduo é o seu próprio controle, o que seria mais apropriado para o seguimento clínico e, no segundo, o indivíduo é comparado com a população geral, mais apropriado para diagnóstico. Como o teste cardiopulmonar do exercício não deve ser considerado isoladamente, ele poderia indicar ao médico assistente um caminho para investigações clínicas adicionais no sentido de apurar o diagnóstico da insuficiência circulatória central ou periférica, de acordo 
com o quadro clínico do paciente em estudo, uma vez que nem à obesidade e nem ao sedentarismo poderia ser atribuída uma porcentagem abaixo de 40,0\% $\%^{2,14,34-37}$. Observaram, ainda, que pacientes classificados como atividade física, ou seja, indivíduos ativos, não sofreriam influência de uma ou outra forma de classificação, que coincide com um grupo na faixa de porcentagem do consumo de oxigênio do limiar anaeróbio em relação tanto ao consumo máximo de oxigênio previsto ou atingido de 50,0 a 59,0\%, indicando que indivíduos saudáveis apenas ativos poderiam ser classificados das duas formas.

\section{Limitações}

O número de pacientes em estudo, a diversidade da apresentação clínica e diagnósticos basais, associado a uma análise descritiva, de um estudo retrospectivo de casos, como relato de experiência, constitui-se certamente na limitação mais importante deste estudo.

\section{CONCLUSÃO}

Os resultados obtidos em um laboratório privado são comparáveis aos publicados pelos grandes laboratórios de exercício, sugerindo que podem ser reproduzidos por outros centros que estão iniciando esta metodologia no país.

\section{REFERÊNCIAS}

1. Sue DY, Wasserman K. Impact of integrative cardiopulmonary exercise testing on clinical decision making. Chest 1991;99:981-92.

2. Neuberg GW. Cardiopulmonary exercise testing. An idea whose time has almost come. Chest 1990;97:257-60.

3. Wasserman K, Hansen JE, Sue DY, Casaburi R, Whipp BJ. Principles of exercise testing and interpretation. Including pathophysiology and clinical applications. $3^{\text {rd }}$ ed. Baltimore: Lippincott Williams \& Wilkins, 1999.

4. Ellestad MH. Stress testing: principles and practice. $4^{\text {th }}$ ed. Philadelphia: F. Davis Co, 1996.

5. Wasserman K. Exercise gas exchange in heart disease. New York: Futura Publishing, 1996.

6. Myers JN. Essentials of cardiopulmonary exercise testing. Illinois: Human Kinetics, 1996.

7. Zavala DC, Mazzei JA. Manual de pruebas de ejercicio y reabilitación cardíaca y pulmonar. Buenos Aires: Centro Editor de la Fundación Favaloro, 1996.

8. Franklin BA, Gordon S, Timmis G. Exercise in modern medicine. Baltimore: Williams \& Wilkins, 1989.

9. Rankin SL, Briffa TG, Morton AR, Hung J. A specific activity questionnaire to measure the functional capacity of cardiac patients. Am J Cardiol 1996;77:1220-3.

10. Palange $\mathrm{P}$, Carlone $\mathrm{S}$, Forte $\mathrm{S}$, et al. Cardiopulmonary exercise testing in the evaluation of patients with ventilatory vs circulatory causes of reduced exercise tolerance. Chest 1994;105:1122-6.

11. Messner-Pellerc P, Ximenes C, Brasileiro CF, et al. Cardiopulmonary exercise testing determinants of dyspnea due to cardiac or pulmonary limitation. Chest 1994;106:354-60.

12. Mahler DA, Horowitz MB. Clinical evaluation of exertional dyspnea. Clin Chest Med 1994;15:259-69.
13. Martinez FJ, et al. Graded comprehensive cardiopulmonary exercise testing in the evaluation of dyspnea unexplained by routine evaluation. Chest 1994;105:168-74.

14. Neuberg GW, Friedman SH, Weiss MB, Herman MV. Cardiopulmonary exercise testing. The clinical value of gas exchange data. Arch Intern Med 1988;148:2221-6.

15. Weber KT, Janicki JS, MacElroy P, Reddy H. Concepts and applications of cardiopulmonary exercise testing. Chest 1988;93:843-7.

16. Mancini D, Eisen H, Kussmaul W, et al. Value of peak exercise oxygen consumption for optimal timing of cardiac transplantation of ambulatory patients with heart failure. Circulation 1991;83:778-86.

17. Brooks GA, Fahey TD, White TP. Exercise physiology - Human bioenergetics and its applications. $2^{\text {nd }}$ ed. Mayfield Publishing, 1995.

18. McArdle WD, Katch FI, Katch VL. Fisiologia do exercício - Energia, nutrição e desempenho humano. 4를. ed. ABDR, Guanabara Koogan, 1996.

19. Wajngarten M, Yazbeck P Jr, et al. Avaliação cardiorrespiratória ao exercício no idoso sadio. Arq Bras Cardiol 1994;63:27-33.

20. Yazbeck PJr, Diament J, Haebish H, et al. Ergoespirometria como método de predição do comportamento evolutivo da miocardiopatia isquêmica, chagásica e idiopática. Arq Bras Cardiol 1991;57:451-8.

21. Yazbeck PJr, Batistella LR. Do atleta ao transplantado: condicionamento físico. São Paulo: Sarvier APM, 1994.

22. Neder JA, Nery LE, Castelo A, et al. Prediction of metabolic and cardiopulmonary responses to maximum cicle ergometry: a randomised study. Eur Resp J 1999;14:1304-13.

23. Novitsky S, Segal KR, Chart-Aryamontri B, et al. Validity of a new portable indirect calorimeter: the aerosport TEM 100. Eur J Appl Physiol 1995;70:104-6.

24. Wideman L, Stoudemire NM, Pass KA, et al. Assessment of the aerosport Teem 100 portable metabolic measurement system. Med Sci Sports Exerc1986;28:509-15.

25. Beaver WL, Wasserman K, Whipp BJ. A new method for detecting the anaerobic threshold by gas exchange. J Appl Physiol 1986;60:2020-7.

26. Borg G. Perceived exertion as an indicator of somatic stress. Scand J Rehabil Med 1970;2:92-8.

27. Froelicher V, Myers J. Exercise and the heart. $4^{\text {th }}$ ed. WB Saunders, 2000.

28. ACC/AHA Guidelines for exercise testing: a report of the American College of Cardiology/American Heart Association Task Force on Practice Guidelines (Committee on Exrcise Testing). J Am Coll Cardiol 1997; 30:260-315.

29. Consenso Nacional de Ergometria - Departamento de Ergometria e Reabilitação Cardiovascular da Sociedade Brasileira de Cardiologia. Arq Bras Cardiol 1995;65:189-211.

30. Serra CM. Infarto agudo de miocardio - Opciones diagnosticas y terapéuticas actuales. Editorial Atlante Argentina SRL, 1994.

31. Braunwald E. Heart disease: a textbook of cardiovascular medicine. $5^{\text {th }}$ ed. WB Saunders, 1997.

32. Buchfuher MJ, Hansen JE, Robinson TE, Sue DY, Wasserman K, Whipp BJ. Optimizing the exercise stress test (or cardiopulmonary assessment). J Appl Physiol 1983;55:1558-64.

33. Higginbotham MB, Morris KG, et al. Physiologic basis for the age-related decline in aerobic work capacity. Am J Cardiol 1986;57:1374-9.

34. Zeballos RJ, Weisman IM. Behind the scenes of cardiopulmonary exercise testing. Clin Chest Med 1994;15:193-213.

35. Weisman IM, Zeballos RJ. An integrated approach to the interpretation of cardiopulmonary exercise testing. Clin Chest Med 1994;15:421-46.

36. McKelvie RS, Jones NL. Cardiopulmonary exercise testing. Clin Chest Med 1989;10:277-91.

37. Weber KT, Janicki JS, McElroy DA, Maskin CS. Cardiopulmonary exercise testing in clinical practice. Cardiology 1987;74:62-70. 